\title{
Multidetector Computed Tomography Imaging for the Diagnosis of Hyperthyroid Cardiomyopathy
}

\author{
Hye Rin Kim, ${ }^{1}$ Seung Min Yoo, ${ }^{1,}{ }^{*}$ Hwa Yeon Lee, ${ }^{2}$ Jae Youn Moon, ${ }^{3}$ Woo In Yang, ${ }^{3}$ and Charles S. White ${ }^{4}$ \\ ${ }^{1}$ Department of Diagnostic Radiology, CHA University College of Medicine, Bundang, Korea \\ ${ }^{2}$ Smile Radiologic Clinic, Seoul, Korea \\ ${ }^{3}$ Department of Cardiology, CHA University College of Medicine, Bundang, Korea \\ ${ }^{4}$ Department of Diagnostic Radiology, University of Maryland Medical Center, Baltimore, USA \\ "Corresponding author: Seung Min Yoo, Department of Diagnostic Radiology, CHA University College of Medicine, Bundang, Korea. Tel: +82-317485423, E-mail: \\ smyoo68@hanmail.net
}

Received 2015 July 26; Revised 2015 September 01; Accepted 2015 September 07.

\begin{abstract}
We present two cases of hyperthyroid cardiomyopathy (CMP) caused by Graves' disease in which multidetector computed tomography (MDCT) findings provided specific diagnostic clues, guiding the attending physician to a prompt diagnosis and allowing rapid initiation of therapy. We believe that a combination of diffuse thyroid enlargement, with decreased attenuation on non-enhanced chest $\mathrm{CT}$, thymic hyperplasia and cardiomegaly (i.e. predominantly right heart failure with enlargement of the left atrium) are suspicious CT findings of hyperthyroid CMP.
\end{abstract}

Keywords: Graves' Disease, Cardiomyopathy, Multidetector Computed Tomography, Hyperthyroidism

\section{Introduction}

Diagnosis of hyperthyroid cardiomyopathy (CMP) has been mainly established on the basis of clinical and laboratory findings (1-5). However, as the field of view of routine chest computed tomography (CT) includes both thyroid glands and heart, multidetector CT (MDCT) has a potential to provide important clues to the presence of hyperthyroid CMP, even if clinical information is not available. We present two cases of hyperthyroid CMP, in which MDCT findings suggested the diagnosis, prior to detailed clinical work-up.

\section{Case Presentation}

\subsection{Case 1}

A 47-year-old male presented with exertional dyspnea for one month. The patient had a history of heavy alcohol intake. However, he had stopped drinking approximately one year earlier. Atrial fibrillation was noted on electrocardiogram (ECG) and chest posteroanterior X-ray showed cardiomegaly and a right pleural effusion. Chest CT was performed for further evaluation. Diffuse thyroid enlargement (diameter of thyroid lobe $=41 \mathrm{~mm}$ and isthmic portion $=10 \mathrm{~mm}$ ) with decreased attenuation (i.e., loss of normal iodine-based high attenuation in the thyroid glands) was noted on non-enhanced chest CT (LightSpeed VCT, GE Healthcare, Milwaukee, WI, USA) (Figure 1A and 1B). The Hounsfield units (HU) of the thyroid glands were markedly lower than normal (HU =71) (Figure 1B). Mild thymic hyperplasia $(53 \times 38 \times 13 \mathrm{~mm})$, bilateral pleural effusions and cardiomegaly [notably of the left atrium (43 mm), main pulmonary artery $(34 \mathrm{~mm})$, right atrium $(56 \mathrm{~mm})$ and right ventricle $(54 \mathrm{~mm})$ ] were noted on contrast enhanced chest CT (Figure 1C-1E ). We notified the attending physician of our high suspicion of hyperthyroid CMP, based on the CT findings, and recommended him to perform thyroid function test, auto-antibody tests and echocardiography. Moderate pulmonary hypertension (right ventricular systolic pressure $=55.3 \mathrm{mmHg}$ ) and grade 2 tricuspid regurgitation were noted on the echocardiography performed the next day. Thyroid function and auto-antibody tests were consistent with Graves' disease [triiodothyronine (T3) $=3.28$ $\mathrm{ng} / \mathrm{mL}$ (normal range, $0.6-1.81 \mathrm{ng} / \mathrm{mL}$ ), thyroid stimulating hormone $(\mathrm{TSH}) \leq 0.01 \mathrm{uIU} / \mathrm{mL}$ (normal range, 0.35 - 5.5 $\mathrm{uIU} / \mathrm{mL}$ ), thyroxine $(\mathrm{T} 4)=7.03 \mathrm{ng} / \mathrm{mL}$ (normal range, 0.89 $1.76 \mathrm{ng} / \mathrm{mL}$ ), thyroid stimulating antibody = positive $(320.3$ $\%$, antithyroid peroxidase antibody (ATPO) $=116.6 \mathrm{IU} / \mathrm{mL}$ (normal range, 5 - $13.6 \mathrm{IU} / \mathrm{mL}$ ), thyroglobulin antibody = $314.6 \mathrm{IU} / \mathrm{mL}$ (normal range, 10 - $124.2 \mathrm{IU} / \mathrm{mL}$ ), TSH receptor antibody $(\mathrm{TRAb})>40.00 \mathrm{U} / \mathrm{L}(0-1.75 \mathrm{U} / \mathrm{L})]$. The therapeutic regimen consisted of $5 \mathrm{mg}$ methimazole administered twice daily. Right ventricular systolic pressure decreased to $38.7 \mathrm{mmHg}$ on follow-up echocardiography performed 2 days after the initial study. Right atrial and ventricular size were also slightly decreased, suggesting interval improvement of pulmonary hypertension, in response to methimazole administration. The patient was ultimately dis- 
charged without dyspnea.

\subsection{Case 2}

A 48-year-old female presented with intermittent chest pain and exertional dyspnea, for 2 months. Sinus tachycardia was identified on ECG. Echocardiography showed moderate pulmonary hypertension (right ventricular systolic pressure $=55.7 \mathrm{mmHg}$ ) and mild tricuspid regurgitation. Chest CT was performed to evaluate the cause of unexplained pulmonary hypertension. The CT features of the patient were approximately similar to those of Case 1 (Figure 2), leading to a high suspicion of hyperthyroid CMP. Thyroid function and auto-antibody tests were consistent with Graves' disease [T3 $=4.39 \mathrm{ng} / \mathrm{mL}$ (normal range, 0.6 - $1.81 \mathrm{ng} / \mathrm{mL}$ ), TSH $\leq 0.01 \mathrm{uIU} / \mathrm{mL}$ (normal range, 0.35 - 5.5 $\mathrm{uIU} / \mathrm{mL}$ ), $\mathrm{T} 4=4.94 \mathrm{ng} / \mathrm{ml}$ (normal range, $0.89-1.76 \mathrm{ng} / \mathrm{mL}$ ), thyroid stimulating antibody $=$ positive $(659.2 \%)$, ATPO $=$ 89.4 IU/mL (normal range, 5 -13.6 IU/mL), thyroglobulin antibody $=183.3 \mathrm{IU} / \mathrm{mL}$ (normal range, $10-124.2 \mathrm{IU} / \mathrm{mL}$ ), TRAb $>40.00 \mathrm{U} / \mathrm{L}(0-1.75 \mathrm{U} / \mathrm{L})$ ]. Therapy with $5 \mathrm{mg}$ methimazole twice daily was started and the patient was discharged without residual symptoms.

\section{Discussion}

There is no prior report in the English literature regarding specific MDCT findings of hyperthyroid CMP. In our cases, diagnostic indicators of hyperthyroid CMP on CT were a combination of the following: diffuse thyroid enlargement with decreased attenuation on non-enhanced chest CT (i.e., loss of normal iodine-based high attenuation of thyroid glands), thymic hyperplasia and cardiomegaly [i.e., predominantly right heart failure (enlargement of main pulmonary artery, right atrium and right ventricle) and left atrial enlargement]. We believe that the combination of the above CT findings is suggestive for the diagnosis of hyperthyroid CMP.

The previous studies reported that decrease in the attenuation of the thyroid glands on non-enhanced CT scan may be related to changes in the metabolism of thyroid hormone in patients with Graves' disease $(6,7)$. Specifically, this may be caused by the fact that in patients with Graves' disease, once iodine is taken up from the blood stream and synthesized to thyroid hormone, iodine is secreted again, without entering the thyroid pool (6). In a previous study, CT density of thyroid gland on the nonenhanced CT in patients with normal thyroid function (122 $\pm 18 \mathrm{HU}$; the lowest value $=87 \mathrm{HU}$ ) was significantly different from that of Grave's disease $(85 \pm 22 \mathrm{HU}, \mathrm{P}<0.001)$ (6). On average, $52.7 \%$ of patients with Grave's disease had a CT density of less than $87 \mathrm{HU}$ in the study. Therefore, loss of normal iodine-based high attenuation in the thyroid glands is expected to be quite specific, even though it is not sensitive as a finding for the detection of hyperthyroidism caused by Graves' disease. Further research is required to address this issue.

Thymic hyperplasia is an additional CT indicator of hyperthyroid CMP in patients with hyperthyroidism (8-10). Thyroid hormone may have a direct effect on the thymus, leading to hyperplasia of thymic cells (8). However, the percentage of thymic hyperplasia on CT, occurring in patients with Grave's disease, has not been elucidated yet and requires further study.

As for the third important CT feature of hyperthyroid CMP, previous studies have indicated that pulmonary hypertension is frequent in patients with hyperthyroidism (11-13). Consequently, hyperthyroid CMP is characterized by CT findings of pulmonary hypertension and predominantly right heart failure (i.e., enlargement of the right atrium, right ventricle, and main pulmonary artery on CT). Several possible mechanisms may account for hyperthyroid-induced pulmonary hypertension: first, thyroid hormones may cause increase in pulmonary vascular resistance, based on a direct effect on pulmonary vasculature (10); second, presence of atrial fibrillation and in crease in blood volume in patients with hyperthyroidism may result in left atrial enlargement. The increase in left atrial pressure leads to the elevation of pulmonary capillary pressure, and, secondarily, an increase in pulmonary arterial pressure. Third, the left ventricular hypertrophy and reduced left ventricular contractile reserve may cause impaired left ventricular filling, resulting in increased left atrial pressure (4). Therefore, hyperthyroid CMP should be suspected in patients with unexplained pulmonary hypertension, as described in Case 2. Relatively rapid improvement of pulmonary hypertension after administration of anti-thyroid agent, as in Case 1, may be due to alleviation of atrial fibrillation and decreased pulmonary arterial resistance.

In conclusion, diagnosis of hyperthyroid CMP is possible based on recognition of certain MDCT findings. Familiarity with CT features of hyperthyroid CMP can lead to prompt diagnosis, early treatment and a better clinical outcome.

\section{Acknowledgments}

None.

\section{Footnotes}

Authors' Contributions: Study concept and design: Hye Rin Kim and Seung Min Yoo; acquisition of data, Hye Rin 

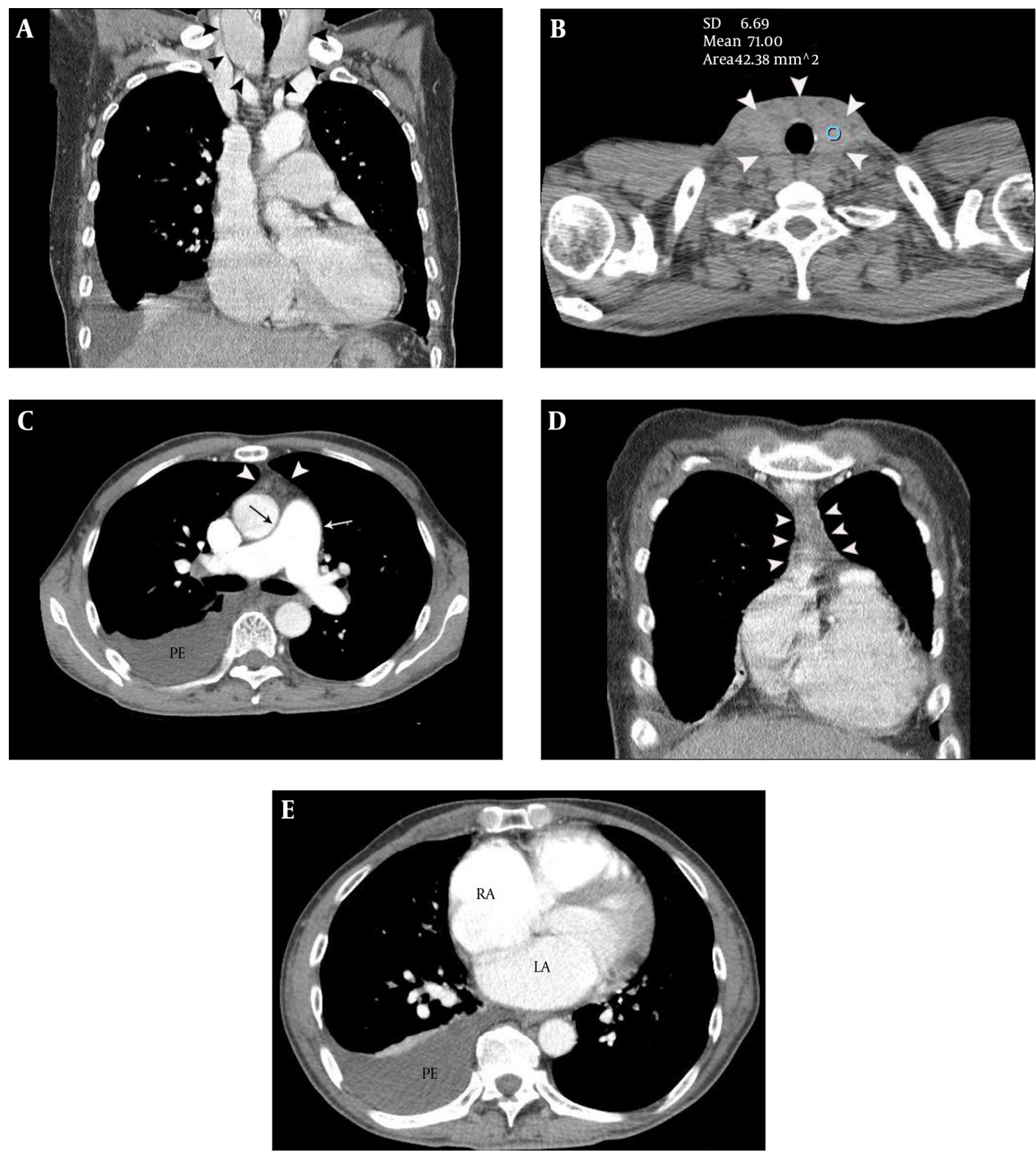

Figure 1. A 47-year-old male with exertional dyspnea and atrial fibrillation. A and B, Diffuse thyroid enlargement (arrowheads on A and B) with a decreased attenuation (HU = 71) was noted on non-enhanced chest CT. Note the loss of the normal high attenuation in the thyroid glands (B). C, Mild thymic hyperplasia (arrowheads) and enlargement of main pulmonary artery (arrows) are demonstrated on contrast enhanced chest CT, at the level of bifurcation of the main pulmonary artery. Right pleural effusion is also noted. D, Mild thymic hyperplasia (arrowheads) is evident on a coronal CT image. E, Enlargement of the left and right atria and right pleural effusion are demonstrated on contrast enhanced chest CT, at the level of the left atrium.

Kim, Seung Min Yoo, Hwa Yeon Lee, Jae Youn Moon, Woo In Yang, Charles S White; analysis and interpretation of data, Hye Rin Kim, Seung Min Yoo, Hwa Yeon Lee, Jae Youn Moon, Woo In Yang, Charles S White; drafting of the manuscript: Hye Rin Kim, Seung Min Yoo, Hwa Yeon Lee, Jae Youn Moon, Woo In Yang, Charles S White; critical revision of the manuscript for important intellectual content, Hye Rin
Kim, Seung Min Yoo, Hwa Yeon Lee, Jae Youn Moon, Woo In Yang,Charles S White; statistical analysis, not available (this is case report); administrative, technical, and material support, Hye Rin Kim, Seung Min Yoo, Hwa Yeon Lee, Jae Youn Moon, Woo In Yang, Charles S White; study supervision, Seung Min Yoo.

Financial Disclosure: None declared. 

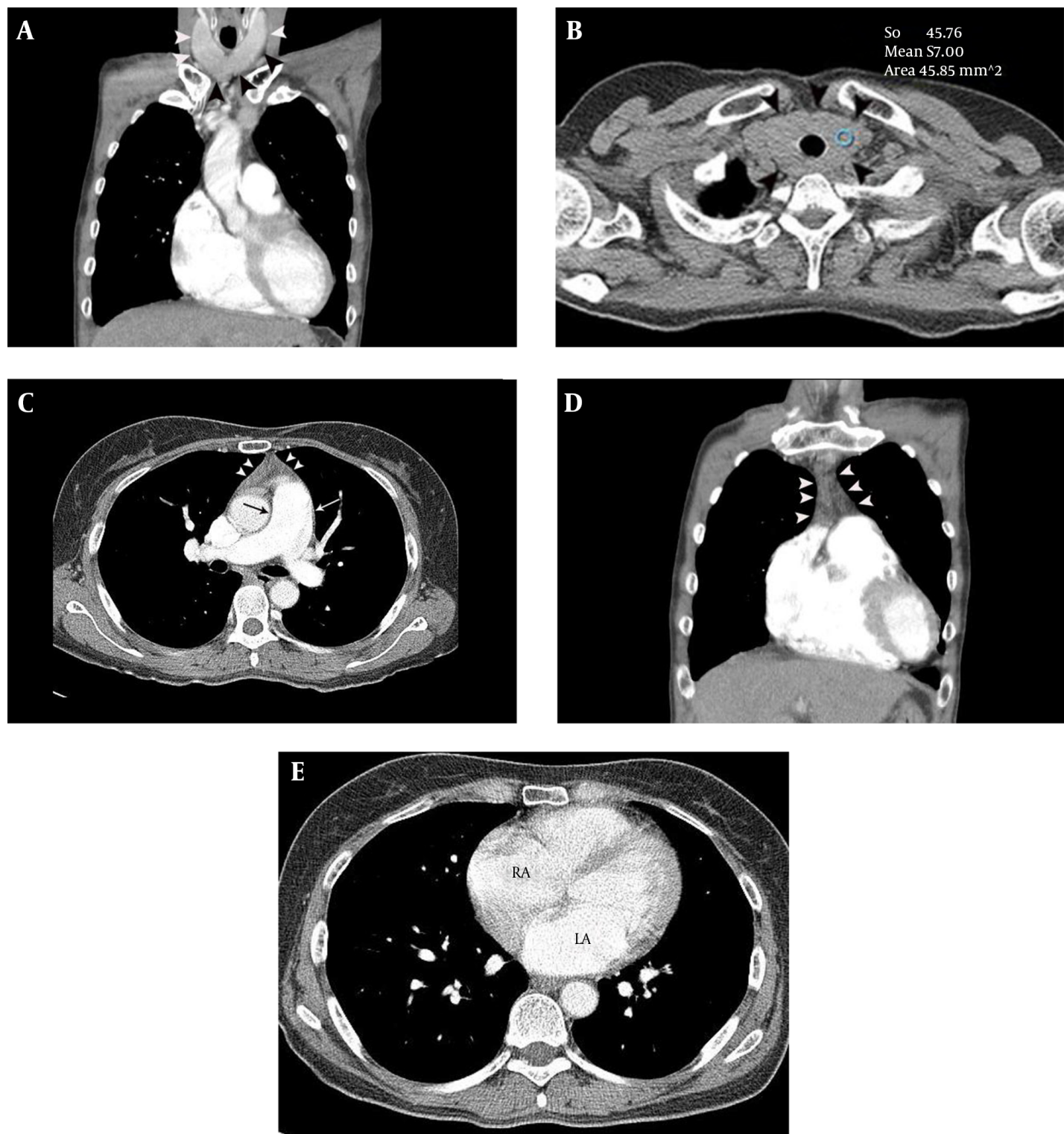

Figure 2. A 48-year-old female with intermittent chest pain, exrtional dyspnea, sinus tachycardia and moderate pulmonary hypertension. A and B, Diffuse thyroid enlargemen (diameter of thyroid lobe $=27 \mathrm{~mm}$ and isthmic portion $=12 \mathrm{~mm}$ ) (arrowheads on A and B) with a decreased attenuation (HU = 57) was noted on non-enhanced chest CT (B). $\mathrm{C}$, Mild thymic hyperplasia (arrowheads) and mild enlargement of main pulmonary artery $(32 \mathrm{~mm})$ (arrow) are demonstrated on contrast enhanced chest CT, at the level of bifurcation of the main pulmonary artery. D, Mild thymic hyperplasia $(39 \times 47 \times 13 \mathrm{~mm})$ (arrowheads) is evident on a coronal CT image. E, Enlargement of the left ( $44 \mathrm{~mm}$ ) and right atrium $(54 \mathrm{~mm})$ is demonstrated on contrast enhanced chest $\mathrm{CT}$, at the level of the left atrium.

\section{Funding/Support: None declared.}

\section{References}

1. Hoang JK, Sosa JA, Nguyen XV, Galvin PL, Oldan JD. Imaging thyroid disease: updates, imaging approach, and management pearls. $R a$ diol Clin North Am. 2015;53(1):145-61. doi: 10.1016/j.rcl.2014.09.002. [PubMed: 25476178].

2. Vaidya B, Pearce SH. Diagnosis and management of thyrotoxicosis. BMJ. 2014;349:5128. doi: 10.1136/bmj.g5128. [PubMed: 25146390].
3. Klein I, Ojamaa K. Thyroid hormone and the cardiovascular system. N Engl J Med. 2001;344(7):501-9. doi: 10.1056/NEJM200102153440707. [PubMed: 11172193].

4. Klein I, Danzi S. Thyroid disease and the heart. Circulation 2007;116(15):1725-35. doi: 10.1161/CIRCULATIONAHA.106.678326. [PubMed: 17923583].

5. Paran Y, Nimrod A, Goldin Y, Justo D. Pulmonary hypertension and predominant right heart failure in thyrotoxicosis. Resuscitation. 2006;69(2):339-41. doi: 10.1016/j.resuscitation.2005.09.002. [PubMed: 16519987]. 
6. Kamijo K. Clinical studies on thyroid CT number in Graves' disease and destructive thyrotoxicosis. Endocr J. 1994;41(1):25-30. [PubMed: 7951548].

7. Han YM, Kim YC, Park EK, Choe JG. Diagnostic value of CT density in patients with diffusely increased FDG uptake in the thyroid gland on PET/CT images. AJR Am J Roentgenol. 2010;195(1):223-8. doi: 10.2214/AJR.09.3319. [PubMed: 20566820].

8. Hamzaoui AA, Klii RR, Salem RR, Kochtali II, Golli MM, Mahjoub SS. Thymic hyperplasia in a patient with Grave's disease. Int Arch Med. 2012;5:6. doi:10.1186/1755-7682-5-6. [PubMed: 22321290].

9. Yacoub A, Gaitonde DY, Wood JC. Thymic hyperplasia and Graves disease: management of anterior mediastinal masses in patients with Graves disease. Endocr Pract. 2009;15(6):534-9. doi: 10.4158/EP09025.ORR. [PubMed: 19491074].

10. Carvalho MR, Dias T, Baptista F, do Carmo I. Graves' disease and massive thymic hyperplasia. Thyroid. 2010;20(2):227-9. doi: 10.1089/thy.2009.0251. [PubMed: 20151833].

11. Osman F, Franklyn JA, Holder RL, Sheppard MC, Gammage MD. Cardiovascular manifestations of hyperthyroidism before and after antithyroid therapy: a matched case-control study. J Am Coll Cardiol. 2007;49(1):71-81. doi: 10.1016/j.jacc.2006.08.042. [PubMed: 17207725].

12. Marvisi M, Zambrelli P, Brianti M, Civardi G, Lampugnani R, Delsignore R. Pulmonary hypertension is frequent in hyperthyroidism and normalizes after therapy. Eur J Intern Med. 2006;17(4):267-71. doi: 10.1016/j.ejim.2005.11.023. [PubMed: 16762776].

13. Sugiura T, Yamanaka S, Takeuchi H, Morimoto N, Kamioka M, Matsumura Y. Autoimmunity and pulmonary hypertension in patients with Graves' disease. Heart Vessels. 2015;30(5):642-6. doi: 10.1007/s00380-014-0518-3. [PubMed: 24838983]. 